Www.jmscr.igmpublication.org

Index Copernicus Value: 79.54

ISSN (e)-2347-176x ISSN (p) 2455-0450

crossref DOI: https://dx.doi.org/10.18535/jmscr/v7i4.143

Original Article

\title{
Role of Sentinel Lymph Node Biopsy and Axillary Node Sampling as a Prognostic Marker in Breast Cancer
}

\section{Rajendra Singh ${ }^{1}$, Ranvijay Bharti ${ }^{2 *}$, Rajshekhar ${ }^{3}$}

${ }^{1}$ M.S (Surgery), Associate Professor, Department of Surgery, Vardhman Institute of Medical Science, Nalanda Bihar, India

Email: Rajendra.singh389@gmail.com

${ }^{2}$ M. S (Surgery), $2^{\text {nd }}$ yr. PG student, Department of Surgery, Nalanda Medical College, Patna, Bihar, India

${ }^{3}$ M.S (Surgery), Senior Resident, Department of Surgery, Nalanda Medical College, Patna, Bihar, India

*Corresponding Author

Dr Ranvijay Bharti

M.S (Surgery), $2^{\text {nd }}$ year PG. Student, Department of Surgery, Flat no 5, block 1, New PG doctors hostel, IDH Colony, Agamkuan, Nalanda Medical College, Patna, Bihar, India.

Email:drranvijay@gmail.com

\begin{abstract}
This study aims to assess whether a combination of sentinel lymph node biopsy (SLNB) using patent blue dye and axillary node sampling (ANS) offers equivalent identification rate to dual tracer technique. Furthermore, we aim to investigate whether there are any potential benefits to this combined technique. Retrospective study of 250 clinically node-negative patients undergoing breast conserving surgery for single T1-T3 tumours between April 2017 and not 2018. Axillary lymph node were staged using a combined blue dye SLNB/ANS technique. SLNs were localized in 245/250 (identification rate $98 \%$ ). Three of one hundred ninety patients with a negative SLN were found to have positive ANS nodes and 1/4 failed SLNB patients had positive ANS nodes. Forty of two hundred thirty five patients had SLN metastases and 13/40(32.5\%) also had a positive non-sentinel lymph node on ANS. Twenty two of twenty five (88\%) node positive. T1 tumours had single node involvement. Ten of forty node-positive patients progressed to complete axillary clearance $(c A L N D)$ and the rest were treated with axillary radiotherapy. Axillary recurrence was nil at median 5 year follow-up. Complementing SLNB with axillary node sampling (ANS) decreases the unavoidable false negative rate associated with SLNB. Appropriate operator experience and technique can result in an SLN localization rate of $98.5 \%$..The additional insight offered by ANS into the status of non-sentinel nodes has potential applications in an era of less frequent cALND.

Keywords: Sentinel lymph node biopsy, Breast cancer, Axillary node clearance and Axillary node sampling.
\end{abstract}

\section{Introduction}

The status of the axillary lymph nodes is a vital prognostic marker in breast cancer management. Traditionally, a complete axillary lymph node dissection (cALND) constituted the gold standard in axillary surgery; however, associated morbidity and the emergence of sentinel lymph node biopsy (SLNB), as a validated staging investigation in breast cancer, have led to reservation of cALND as a follow on therapeutic procedure for patients with positive sentinel lymph nodes (SLNs). 
Following the acceptance of SLNB as a standard of care, increased research has been conducted to consider the significance of a positive SLNB, whether this be macro metastases, micro metastases or isolated tumour cells and the likelihood of further positive axillary nodes. Predicting the likelihood of further positive SLN would allow an individualized approach by targeting patients who will benefit from further therapeutic axillary interventions in the form of surgery or radiotherapy. Various prediction models have been described as well as novel imaging techniques. These have not been translated into routine clinical practice. A new question under consideration is whether there is sufficient evidence to implement towards nonsurgical management and avoid the use of cALND in cases of 'early' clinically node negative breast cancer who have low nodal disease burden on SLNB.

\section{Background}

Sentinel lymph node biopsy is generally regarded as a safe and accurate technique for axillary staging. A number of cohort studies and subsequent randomised control trials have demonstrated a comparable overall survival, disease free survival and regional recurrence with routine axillary dissection.

The false negative rate of SLNB has been shown to range from 0 to $10.5 \%$; however, decreasing rates have been reported with increased operator experience. Both the American Society of Clinical Oncology (ASCO) and the National Institute of Health and Clinical Excellence (NICE) recommend using a minimally invasive approach with SLNB in staging clinically negative axillary lymph nodes, preferably using a dual tracer(blue dye and radioisotope) technique.

Axillary four lymph node sampling is another less invasive axillary staging technique, which comprises identification via direct palpation and excision of four nodes from level 1.

This study aims to assess whether a combination of SLNB using patent blue dye and axillary node sampling (ANS) offers equivalent identification rate to dual tracer technique. Furthermore, we aim to investigate whether there are any potential benefits to this combined technique.

\section{Methods}

The study group comprised 250 consecutive patients treated with breast conserving surgery (BCS) performed by a single surgeon between 2017 and 2019. All patients presented with clinically node negative, single tumour, breast cancers stage T1a to T3. Axillary lymph nodes were assessed with a combination of clinical and ultrasound examination. Ultrasound guided fine needle aspiration cytology of lymph nodes was introduced in 2018. Both palpable and impalpable breast lesions were included; impalpable tumours were identified preoperatively via fine wire localisation and/or US black dot marking. Most procedures were performed as day cases. Neither breast nor axillary drains were used.

A single tracer technique was used to identify the SLN. Following induction of general anaesthesia, $2 \mathrm{~mL}$ of blue tracer dye (sodium patent blue V 2.5 $\%$ injection, Laboratoire Guerbet, Villepinte, France) was injected under the dermis of the areola in the quadrant corresponding with the tumur location.

That area was briefly massaged for approximately $1 \mathrm{~min}$. Ten minutes following injection the axilla was accessed using a low axillary skin crease incision. All blue stained lymph nodes were excised and labelled as sentinel nodes. Identification and excision of further lymph nodes from level 1, via direct palpation or visualization, comprised the axillary sampling (ANS), until minimum four lymph nodes were excised.

Histopathological examination of specimens was performed according to RC Path UK guidelines. Specimens were embedded and processed in $2 \mathrm{~mm}$ thick slices with haematoxylin and eosin staining. Conventional microscopic examination of haematoxylin and eosin (H\&E) stained tissue sections was performed. 
Each case was subsequently discussed at the weekly multidisciplinary team meeting. Although regional guidance prior to 2017 for the study unit was to consider cALND with any evidence of micro or macro metastases following SLNB, cALND was usually reserved for patients with positive nodal disease and T3, grade 3,ER-negative or HER2-positive tumours. Adjuvant radiotherapy was given within 6-8 weeks following surgery in accordance with dosage recommendations provided by the North of England Cancer Network. Patients requiring no therapeutic axillary intervention received isocentric 3D planned tangential field whole breast irradiation of $40 \mathrm{~Gy}$ in 15 divided fractions over 3 weeks. Women under the age of 40 or $>40$ years old with a resection margin of $<3 \mathrm{~mm}$ were given a tumour bed boost of 10 Gy in five fractions over a week. Titanium clips were placed at the time of surgery in the excision bed to ensure accurate delivery of the radiotherapy boost. Patients with one or two positive sentinel or axillary sampling nodes (micro or macro metastases) were given levels I and II axillary field as well as whole breast irradiation. This consisted of 50 Gy in 25 fractions over 5 weeks. Adjuvant endocrine therapy in the form of letrozole $2.5 \mathrm{mg}$ once daily was given in ERpositive cases. Adjuvant anthracycline containing polychemotherapy was given if indicated. All patients with HER2-positive breast cancer with tumour size $>1 \mathrm{~cm}$ were referred to the medical/clinical oncologist for discussion of adjuvant chemotherapy followed by trastuzumab (Herceptin).

\section{Results}

All 250 patients were women aged 32-82 years old (mean 60,median 61,SD 8.6);190 patients were identified by the NHS Breast Screening Programme (NHSBSP) and 60 patients were symtotomatic referrals to the outpatient clinic.

Table 1 illustrates patient demographics.

One hundred ninety tumours were stage $\mathrm{T} 1$, fifty T2 and ten T3 (Table 1). SLNs were localized in 245 patients; therefore, the sensitivity of blue dye for SLNB is $98 \%$, which is equitable to the identification rate of $97 \%$ shown by the AMAROS trial using a combination approach of blue dye and radioisotope. Three of one hundred ninety patients with a negative SLN were found to have positive macro metastatic axillary sampling nodes (Table 2). This equates to a false negative rate of $8 \%$ and negative predictive value $98 \%$.One of four patients who failed SLN localisation had positive macro metastatic axillary sampling nodes. Axillary sampling improved staging accuracy in 10/250 patients: 4 patients with negative SLNB had positive axillary sampling nodes and 6 patients who failed SLN identification were still able to undergo axillary staging.

The mean number of SLNs harvested was 2.75 (median 2, range 0-7); mean number of axillary sampling nodes was 4.25 (median 4,range 0-9) and mean number of total nodes sentinel and sampling excised was 6.25 (median 6,range 4-9) and mean number of total nodes excised was higher than intended probably because more than one node can be present in a specimen.

Table 1 Population demographics/tumour characteristics

$\begin{array}{lc}\text { Age } & \\ 30-40 & 5 \\ 41-50 & 25 \\ 51-60 & 88 \\ 61-70 & 110 \\ 71-80 & 20 \\ 80+ & 2 \\ \text { Tumour grade } & \\ \text { Grade I } & 66 \\ \text { Grade II } & 105 \\ \text { Grade III } & 75 \\ \text { Mixed/ungraded } & 4 \\ \text { Tumour stage } & \\ \text { Tla } & 15 \\ \text { Tlb } & 80 \\ \text { Tlc } & 112 \\ \text { T2 } & 35 \\ \text { T3 } & 8 \\ \text { Hormone receptor status } & \\ \text { ER positive } & 190 \\ \text { ER negative } & 50 \\ \text { Unrecorded } & 10 \\ \text { HER2 status } & \\ \text { HER2 positive } & 45 \\ \text { HER2 negative } & 195 \\ \text { Unrecorded } & 10\end{array}$


The mean number of total nodes excised was higher than intended probably because more than one node can be present in a specimen.

Forty five of two hundred fourty five patients with successful SLN localization had SLN metastases (Table 2). Thirteen of forty two (30.95\%) of these patients were also found to have a positive axillary sampling node. In 25/42 (59.52\%) patients with positive SLN, further axillary sampling did not show any additional axillary disease.

Table 2 Distribution of number of positive sentinel lymph nodes

Number of positive sentinel lymph nodes Number of patients

1
2
3

2

Negative

No.of SLN

29

7

2

208

4

\section{Discussion}

Axillary treatment is evolving due to early evidence that cALND may no longer be necessary in clinically node negative $\mathrm{T} 1$ or $\mathrm{T} 2$ tumours with less than three positive sentinel lymph nodes. The 2013 St.Gallen consensus supports avoidance of cALND in clinically node negative patients with 1-2 macro metastatic sentinel nodes operated with breast conservation and receiving tangential field adjuvant radiotherapy. The American College of Surgeons Oncology Group Z0011 trial provides indication that cALND may no longer be clinically justified in T1-2 breast cancer, as low regional recurrence rates were demonstrated with breast conserving surgery, breast radiotherapy and adjuvant systemic therapy alone. The study, which randomly assigned patients with SLN positive breast cancer to SLN biopsy and ALND or to SLN alone, reported that ALND was not associated with any survival benefit and that both groups had an extremely low regional recurrence rate $(0.9 \%$ for SLN alone and 0.5\% for ALND). This was despite the fact that $27 \%$ of the patients who received ALND had additional positive non sentinel lymph nodes.

It is noteworthy that low accrual was a consequence of a lower than expected event rate in both arms. The time necessary to accrue the patients in order to fulfil the power calculation would have taken many years, rendering the trial impractical. Another concern pertains to the type of irradiation patients received and difficulties in ascertaining homogeneity across the group. While direct nodal irradiation was forbidden, the irradiation field could be modified to include low axillary regions in patients who did not have cALND. This could represent a source of bias and explain the low regional recurrence rate in the SLNB group. Axillary nodal disease burden was also low with $40 \%$ patients having micro metastatic disease or isolated tumour cells.

The primary tumour was treated with either mastectomy or breast conserving surgery, with $90 \%$ of patients undergoing BCS. Patients undergoing BCS received a combination of ELIOT intra-operative tumour bed irradiation (28\%) or post-operative tangential field whole breast irradiation. Thirteen percent of cALND patients had atleast one further positive node. Twenty four percent of patients who did not receive cALND either did not have any radiotherapy (4\%) or received ELIOT partial breast irradiation which does not irradiate the axilla (20\%).In spite of this discrepancy, results showed no significant difference in either disease free (DF) or overall survival (OS) at a median of 5 years follow up.

Higher morbidity was observed in the cALND group consistent with previous findings. A recent systematic review proposed to assess the role of axillary interventions in breast cancer treatment with regard to recurrence of axillary node metastases, mortality and morbidity. RCTs needed to have at least 2 years follow up;non-randomized trials needed to have at least 2 years follow up in at least $50 \%$ of patients. Most studies were conducted in patients with clinically impalpable axillary nodes. Treatment modalities varied and included mastectomy, breast conserving therapy, axillary lymph node dissection, whole breast irradiation and tamoxifen. cALND in patients with no palpable lymph nodes compared with sentinel node biopsy gave no survival benefit and was 
associated with a 1 to $3 \%$ reduction in recurrence of axillary node metastases but an increased risk of lymphoedema (14\% compared to 5 to $7 \%$ for biopsy).The authors concluded that there was little evidence of benefit from surgical cALND compared with SLNB alone in patients with clinically node negative breast cancers $<3 \mathrm{~cm}$ who had up to three positive SLNB.

A meta analysis was not undertaken although it may have been possible to do so with inclusion of subgroup analyses for the different interventions. The lack of quality assessment and of statistical pooling renders the conclusions uncertain.

Although the findings of the above mentioned studies have not been adopted into routine clinical practice, the evidence does have important clinical implications. Whilst these studies are open to criticism due to either study design, lack of statistical power, potential for bias or other mitigating factors such as heterogenous adjuvant chemo and radiotherapy regimens, the presence of limiting factors cannot simply discredit the purported concept and emergence of increasing evidence that a subset of patients with SLN metastases may do well without cALND. The issue is how best to target such cases. Several predictive nomograms have been devised but are not accurate enough to warrant clinical application. Novel imaging techniques (e.g.nanoparticle MRI) have not reached clinical maturity. The UK Positive Sentinel Lymph Node: Observation vs Clearance (POSNOC) trial will be a logical sequel to Z0011 and IBCSG trial. It will randomly assign to observation only or CALND both patients who undergo breast conserving surgery and those who undergo mastectomy with one or two sentinel lymph nodes containing macro metastases.

Axillary nodal disease is only a (potentially crude) surrogate marker for tumour behaviour which may be superseded by molecular and DNA profiling in the near future. Our data which predates the publication of the cited studies shows that avoidance of cALND in patients with early (T1T2) breast cancer with 1-2 positive SLN, who are treated with BCS and whole breast/axillary radiotherapy, is safe. None of our patients had axillary recurrence at a median follow-up of 5 years.

Post mastectomy (PMRT) and supraclavicular fossa radiotherapy (SCFRT) depend on nodal status (>4 macro metastatic nodes).A recent report highlighted the potential risk of under staging patients who only have SLNB.A combination approach of blue dye sentinel lymph node biopsy and axillary node sampling (SLNB/ANS) is a potentially useful investigation to estimate the risk of additional positive nodes. Using ANS as an adjunct to SLNB provides additional insight into the status of the non sentinel axillary lymph nodes and increases confidence in the safety of non surgical management of axillae with positive SLN and, as a consequence may be useful in preventing axillary under staging. Appropriate injection and surgical technique using blue dye alone can ensure high detection rates which equal that of large series using dual tracer which has positive implications for countries where radioisotope is not generally available.

\section{Limitations}

The data set is from a single surgeon consecutive case series, although this does have added strength in terms of technical consistency. The single tracer SLN localization is higher than average; therefore, the ability of this study to compare a combined SLNB/ANS technique with a dual tracer technique is limited.

Previous studies have demonstrated clinically significant lymphoedema rates of $19 \%$ at 5 years following cALND compared to $5 \%$ following ANS. Furthermore, non surgically managed node positive axillae received axillary radiotherapy, which has the potential for morbidity, although this is lower than cALND. Data regarding health related quality of life outcomes as a result of the combined SLNB/ANS technique remains observational, with no evidence of increased morbidity compared to sentinel node biopsy alone. An additional study of this patient group is 
underway to consider these outcomes more objectively. Further evidence (i.e. POSNOC trial), verifying the safety of treatment algorithms proposed by the Z0011 and IBCSG trials, may prove that axillary radiotherapy as well as cALND is an unnecessary overtreatment. However, as our data predates the publication of cited trials, nonsurgical management with axillary radiotherapy of patients with 1-2 positive nodes seemed acautious approach at that time.

\section{Conclusion}

Appropriate operator experience and technique can result in an SLN localization rate of $98 \%$, rivalling a dual tracer technique SLNB with axillary node sampling (ANS) decreases the unavoidable false negative rate associated with SLNB and may prove beneficial for less experienced operators. Also, the additional insight offered by ANS into the status of non sentinel nodes has potential applications in an era of less frequent cALND.

\section{References}

1. Krag DN, Anderson SJ,Julian TB et al(2010)Sentinel-lymph- node resection compared with conventional axillarylymph-node dissection in clinically nodenegative patients with breast cancer: overall survival findings from the NSABP B-32 randomised phase 3 trial. Lancet Oncol 11:927-933.doi:10.1016/S14702045(10)70207-2

2. Veronesi U, Paganelli G, Galimberti V et al(1997) Sentinel-node biopsy to avoid axillary dissection in breast cancer with clinically negative lymph-nodes. Lancet 349:1864-1867.doi:10.101S0140-

6736(97)01004-0

3. NICE Breast cancer (early \& locally advanced).In:

NICE.

http://www.nice.org.uk/.Accessed 12 Apr 2014

4. Dordea M,Colvin H,Cox P et al(2013) Clinical and histopathological factors affecting failed sentinel node localization in axillary staging for breast cancer. Surgeon 11:63-66. doi:10.1016/j.surge.2011.10.006

5. Lyman GH, Temin S, Edge SB et al (2014) Sentinel lymph node biopsy for patients with early-stage breast cancer: American society of clinical oncology clinical practice guideline update.J Clin Oncol.doi:10.1200/JCO.2013.54.1177

6. Forrest AP, Everington D,Mc Donald CC et al (1995) n Edinburgh randomized trial of axillary sampling or clearance after $\mathrm{m}$ astectomy.Br J Surg 82:1504-1508

7. Bassi KK,Seenu V,Srivastava A,Al Sharara N(2012)Ro axillary sampling in the era of sentinel Iymph node biopsy:a cnr Of review. Indian J Cancer 49:6673.doi:10.4103/0019-509x osnca

8. Adwani A,Ebbs SR,Burton S, Lowe S (2005) Sentinel node sy should be supplem ented by axillary sampling in patients with small breast cancers.Int Semin Surg Oncol2:27.doi:10.1186/147h 7800-2-27

9. Parmar V,Hawaldar R,Nair NS et al(2013)Sentinel node bions versus low axillary sampling in women with clinically node nenytive operable breast cancer. Breast 2:011086.do10.106breast.2013.06.006

10. Northern England Cancer Network Breast Cancer Clinic Guidelines (2015) http://www.nescn.nhs.uk/wpcontent/uploads/ 2015/02/NECN-BreastCancer-Guidelines-April-2015-2-5.pd11.

11. Straver ME, Meijnen P,van Tienhoven G et al(2010)Sentinel nod identification rate and nodal involvement in the EORTC 10981.22023 AMAROS trial. Ann Surg Oncol 17:1854-1861.doi: 1245/s10434010-0945-

12. Sanuki N,Takeda A,Amemiya A et al(2013)Outcomes of clinically nodenegative breast cancer without axillary dissection: can preserved axilla be safely 
treated with radiation after a positive sentinel node biopsy? Clin Breast Cancer 13:69-76.doi:10.1016j.clbc.2012.09.005

13. Goldhirsch A,Winer EP,Coates AS et al(2013)Personalizing the treatment of women with early breast cancer:highlights of the St Gallen international expert consensus on the prim ary therapy ofearly breast cancer 2013. Ann Oncol.doi:10.1093/annonc/mdt3

14. Giuliano AE,McCall L,Beitsch $P$ et al (2010) Locoregional recur-rence after sentinel lymph node dissection with or without axillary dissection in patients with sentinel lymph node metastases:the American College of Surgeons Oncology Group Z0011 randomized trial. Ann Surg 252:426-

432.doi:10.109/SLA.0b013e3181f0832, dis cussion432-433

15. Lucci A,McCall LM,Beitsch PD et al(2007)Surgical complications associated with sentinel lymph node dissection (SLND) plus axillary lymph node dissection compared with SLND alone in the American College of Surgeons Oncology Group Trial Z0011.JClinOncol 25:3657-

3663.doi:10.1200/JCO.2006.07.4062

16. Latosinsky S,Berrang TS,Cutter CS et al(2012)CAGS and ACS evidence based reviews in surgery.40.Axillary dissection versus noaxillary dissection in women with invasive breast cancer and sentinel node metastasis.Can J Surg 55:6669.doi:10.1503/cjs.036011

17. Giuliano AE,Morrow M,Duggal S,Julian TB(2012)ShouldACOSOG Z0011 change practice with respect to axillary lymph node dissection for a positive sentinel lymph node biopsy in breast cancer? Clin Exp Metastasis 29:687-692. doi:10.1007/s1058 - 012-9515-Z

18. Galimberti V,Cole B,Zurrida $S$ et al(2011)S3-1:update of intemtional breast cancer study group trial 23-01 to compare axillary dlssection versus no axillary dissection in patients with clinically nodenegative breast cancer and micrometastases in the sentinel nodc Cancer Res 71:S3-1.doi:10.1158/00085472.SABCS11-S3-1

19. Galimberti V,Cole BF, Zurrida $S$ et al (2013) Axillary dissecid versus no axillary dissection in patients with sentinelnodd micro metastases (IBCSG 23-01):a phase 3 randomised controla) trial. Lancet Oncol 14:297-305. doi:10,1016/S14702045(70035-4

20. Wernicke AG, Goodman RL,Turner BC et al (2011)A 10-year tollow-up of treatment outcomes in patients with early stage Dicrial cancer and clinically negative axillary nodes treated with tangenxil breast irradiation following sentinel ivmph node dissection O Hary clearance. Breast Cancer Res Treat 125:893-902. doi:10.TOus10549-010-1167-6

21. Houvenaeghel G,Nos C,Mignotte $\mathrm{H}$ et al(2006) Micro metastases in sentinel lymph node in a multicentric study predictive factors of nonsentinel lymph node involvement-Groupe des Chirurgiens dela Federation des Centres de Lutte Contre le Cancer.J Clin Oncol24:18141822.doi:10.12/ 0.2005.03.3225

22. Nos C ,Harding-MacKean C,Freneaux P et al(2003)Prediction of tumour involvement in remaining axillary lymph nodes when the sentinel node in a woman with breast cancer contains metastases.Br J Surg 90:1354-1360.doi:10.1002/bjs.4325

23. Gur AS,Unal B,Johnson R et al(2009) Predictive probability of four different breast cancer nomograms for nonsentinel axillary lymph node metastasis in positive sentinel node biopsy.J Am Coll Surg 208:229-235.doi:10.1016/j jamcollsurg.2008.10.029 
24. Moghaddam Y,Falzon M,Fulford L et al (2010) Comparison of three mathematical models for predicting the risk of additional axillary nodal metastases after positive sentinel lymph node biopsy in early breast cancer.Br J Surg 97:16461652.doi:10.1002/bjs.7181

25. Johnson L, Pinder SE, Douek M (2013) Deposition of superparamagnetic ironoxide nanoparticles in axillary sentinel lymph nodes following subcutaneous injection.Histopathology62:481486.doi:10.1111/his. 12019

26. Albain KS,Barlow WE,Shak S(2010) Prognostic and predictive value of the 21gene recurrence score assay in postmenopausal women with nodepositive, oestrogen-receptor-positive breast cancer on chemotherapy:a retrospective analysis of a random ised trial.Lancet Oncol 11:55-65. doi:10.1016/S14702045(09)70314-6

27. Mamounas EP,Tang G,Fisher B et al(2010)Association between the 21-gene recurrence score assay and risk of locoregional recurrence in node-negative, estrogen receptor-positive breast cancer: results from NSABP B-14 and NSABP B20.J Clin Oncol 28:16771683.doi:10.1200/JCO.2009.23.7610

28. Hussain T,Kneeshaw P(2014)Axillary lymph node clearance in patients with positive sentinel lymph node biopsy. Ann R Coll Surg Engl 96:199-201. doi:10.1308/003588414X13814021678592

29. Tanguay JS,Ford DR,Sadler G et al(2008)Selective axillary node sampling and radiotherapy to the axilla in the management of breast cancer. Clin Oncol(R Coll Radiol)20:677682.doi:10.1016/j.clon. 2008.06.006
30. Chetty U,Jack W,Prescott RJ et al(2000)Management of the axillain operable breast cancer treated by breast conservation:a randomized clinical trial. Edinburgh Breast Unit.Br J Surg 87:163169.doi:10.1046/j.13652168.2000.01345.x 